Audiology

\section{Googly from down under: hearing impairment and early intervention}

\section{K Das, R V Das}

\section{Commentary on the paper by Wake et al (see page 238)}

$\mathrm{T}$ he field of audiological medicine has ridden the wave of technological advancement that has transformed many aspects of screening and intervention in medicine. We can screen newborns for hearing loss, however we are not sure what is the most cost effective and simple method. A screening test that can be referred to as the "golden standard", still eludes us. With the incidence of permanent bilateral clinically significant hearing loss at just over 1 in 1000 births, ${ }^{12}$ the need for a simple, inexpensive, and reliable screening method is evident.

The importance of diagnosing hearing loss at an early stage led to screening originally of "at risk" groups in the form of a targeted screening programme. This approach has proved to be effective as over half of hearing impaired and deaf children can be identified. To identify the other half of hearing impaired children, universal newborn screening programmes are being used in countries including the UK. Various combinations of oto-acoustic emissions and auditory brain stem tests singly or in combination have been successfully tried. Early fitting of technically sophisticated hearing aids and surgical intervention by implantation of cochlear devices is being successfully practised in many centres and countries in both private and state health services.

The practice of both targeted and, more recently, of universal screening, has given us the capability to fit hearing aids very early in infancy. The availability of implanting cochlear devices surgically has also been available and has transformed the management of many cases. The fundamental question "Does this make a difference to the child's development and life?" remains unanswered in many cases, as we still lack a scientific approach to predict the degree of success of early amplification and implantation at an early stage especially during infancy. There are many known variables to take into account, such as additional disabilities—visual impairment, cognitive impairment, genetic and specific learning disabilities. These all have an impact on the outcome of any intervention.

It has been claimed that early diagnosis of hearing impairment and appropriate habilitation leads to a better outcome as seen in improved language and speech development. This simple and logical concept has been propagated since the eminent work by Andreas Markides under the leadership of Professor Ian Taylor from Manchester, UK. ${ }^{3}$ It showed the beneficial effect of early diagnosis and habilitation of children with hearing impairment and deafness. A number of workers followed and reported similar results, but some of them lacked long term follow up and appeared to group together a number of factors influencing the outcome.

Effective management of hearing impairment is a multidisciplinary complex process where the outcome is dominated by a number of factors which may vary in their influential role for an individual child. One of the factors which has clearly been shown to be important in the outcome of a child's habilitation is the degree of hearing impairment. Another widely held belief is that the age of diagnosis is an important determinant of outcome: the notion that the earlier hearing impairment can be identified, the earlier the child can be helped. The article published in this issue of Archives by Wake and colleagues ${ }^{4}$ claims to show that the age of diagnosis was not a relevant factor in dictating outcome as measured by speech and language assessment in this study carried out in Victoria State, Australia. Much like a googly from Shane Warne, this paper takes an unexpected turn away from conventional understanding and other reports in this field. ${ }^{356}$ The study comes to the conclusion that the degree of hearing impairment is the predominant factor influencing the outcome as assessed at the age of 7-8 years.

A weaknesses of this otherwise well constructed study is the small numbers of children, diagnosed early at 6 months of age, in the severe and profound hearing impaired groups. The mean age of diagnosis ( 1.8 years), hearing aid fitting ( 1.9 years), and early intervention programme (2 years) are all later than what would be possible following a universal newborn hearing screening programme. Another problem appears to be a lack of data on high frequency threshold of $4 \mathrm{kHz}$, which may have a crucial role in speech development.

Auro-oral communication and educational approaches to hearing impairment and deaf children has been significantly boosted by early diagnosis and amplification. This has been powered by the unproven notion and concept of a "critical period" of speech and language development related with audition. Wake et al challenge this concept and create debate about the relation of early diagnosis and outcome. Their study points to other known and unknown factors, highlighting the role of motivation and drive, personality, behaviour, auditory processing, and some other innate and unknown factors related with learning that have not been investigated in large follow up studies.

Some of these questions can only be answered by appropriately constructed, prospective, multicentre, large longitudinal studies. More research and auditing of various practices and processes are needed. Neither medical nor educational systems can work independently to answer these questions and a combined effort is needed. There is an ongoing need to concentrate on early diagnosis so that the habilitating processes can be applied and studied. The conclusion of the Wake et al study should not be seen as a case against the need for early diagnosis, but rather a challenge for universal screening. We need to conduct larger well constituted, prospective, multicentre studies to gather detailed information about all factors which may influence outcome. Then, and only then, can we practice evidence based audiology. Studies which highlight the need for further research and that challenge our conceptions are a potent weapon in our further understanding of child healthcare issues and our consequent management strategies.

Arch Dis Child 2005;90:221-222. doi: 10.1136/adc.2004.055137

\section{Authors' affiliations}

V K Das, University Department of Audiological Medicine, Manchester Royal Infirmary, Manchester, UK

R V Das, Medical student, University of Manchester, UK

Correspondence to: $\operatorname{Dr} V \mathrm{~K}$ Das, University Department of Audiological Medicine, Manchester Royal Infirmary, Oxford Road, Manchester M13 9WL, UK; vijay.das@cmmc. nhs.uk 


\section{REFERENCES}

1 Das VK. Aetiology of bilateral sensorineural hearing impairment in children: a 10 year study. Arch Dis Child 1996;74:8-12.

2 Davis A, Bamford J, Wilson I, et al. A critical review of the role of neonatal hearing screening in the detection of congenital hearing impairment. Health Technology Assessmen 1997; $1:(10)$

3 Markides $\mathbf{A}$. Age at fitting of hearing aids and speech intelligibility. Br J Audio 1986;20:165-7.

4 Wake M, Poulakis Z, Hughes EK, et al. Hearing impairment: a population study of age at diagnosis, severity, and language

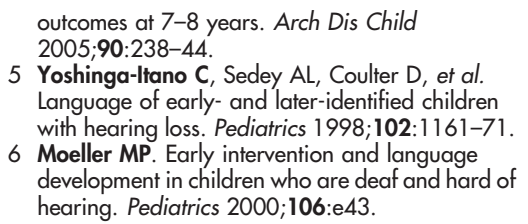

outcomes at 7-8 years. Arch Dis Child with hearing loss. Pediatrics 1998;102:1161-71. development in children who are deaf and hard of hearing. Pediatrics 2000;106:e43.

\section{A deficit that needs attention}

\section{Mather}

\section{Commentary on the paper by Foreman et al (see page 245)}

T he conclusions of the paper by Foreman and colleagues ${ }^{1}$ will not come as a surprise to any parent or paediatrician. Children who behave badly stress their parents. The normal methods of discipline work less well in the face of pervasive hyperactivity, impulsivity, and inattention. After some years of failure, combined with feelings of inadequacy, most parents give up and take the line of least resistance. Paediatricians can do the same. Faced with the chaos of a wrecked surgery and conscious of a very full waiting room, the line of least resistance can suddenly become very attractive. Seeing what appears to be a textbook presentation of a lack of parental discipline, it is all too easy to blame the parents and refer the child on quickly to someone who "does behaviour".

Hyperkinesis is a major risk factor for parenting breakdown, increasing the risk that a child will be excluded from the family by a factor of three. The risk appears to be independent of any other psychosocial difficulties affecting the family. Logically therefore, early detection and treatment might prevent the distress of family breakdown. However, to achieve this laudable aim will not be easy. Hyperkinesis challenges not only our professional tolerance but also the way paediatric services for all community based childhood disorders are currently structured.

"Hyperkinetic disorder", the World Health Organisation term, is the description of choice in Europe. "ADHD; attention deficit, hyperactivity disorder" is the preferred term in America, Australia, and many other parts of the world. Hyperkinetic disorder has stricter diagnostic criteria and therefore applies to fewer children than ADHD. Nevertheless, the figures are striking. One per cent of school aged children in the UK and 3-4\% of school aged children in the USA could be affected. ${ }^{2}$ Foreman et al point out that it is not clear how, or even whether hyperactive children come to the attention of paediatricians in the UK. Hyperkinesis, the commonest, treatable, neuropsychiatric problem of childhood, still sits on the fringes of mainstream paediatric practice. The public health consequences and resource implications of more effective diagnosis, treatment, and parental and educational support for this population of children remain largely unaddressed.

The National Institute for Clinical Excellence Guidelines (NICE) for England and Wales and the Scottish Intercollegiate Guidelines Network (SIGN) in Scotland recommend that all children should be assessed and managed by a specialist. ${ }^{3}{ }^{4}$ Where will the specialists come from to meet the growing demands for assessment and treatment as well as to undertake preventative work? Hyperkinesis is currently the province of the child psychiatrist with an interest (from which many opt out), the community paediatrician with an interest (a diminishing speciality who have taken up the work by default often with little or no mental health training), or (the very occasional) general paediatrician with an interest. Most specialists have very long waiting lists.

Managing hyperkinetic children is neither easy nor quick and does not fit in with a model of healthcare dominated by short waiting times, rapid assessment, intervention, and discharge. The cut-off point between the pathological and a child with a normal but difficult temperament is not clear. Factors in the child's environment, while not causative, do heavily influence the outcome. A child with high scores on a screening questionnaire and two saints for parents attending a small, structured, private school may not need treatment. A child with lower scores and a single harassed parent attending a chaotic large inner city school may. There is still a stigma in being labelled as "hyperactive" in most schools. Many parents, while acknowledging the value of medication, often feel a deep sense of failure compared with the parents of children with other chronic medical problems.

It is not uncommon for specialists, pressurised by teachers, parents, or social workers into prescribing medication, to then be accused by other professionals or family members of succumbing to the temptation of a "chemical straitjacket" to make up for the other social deficits in the child's life. There is not yet a general public or professional consensus that the use of medication might stabilise a family and maintain a child at home or in school.

Assessment is a complicated multidisciplinary process. The most recent national protocol in this journal recommended that an initial assessment should have the following minimal components: a parental interview, a parental rating scale, a child interview, a teacher rating scale, and a descriptive teacher report. The assessment should also document the child's level of academic achievements, and evaluate and record parental attitudes to the child and the child's social relationships with peers and parents. The assessment should include a comprehensive family history, medical and developmental history, and medication history. In addition to a physical examination and height and weight, the child should have hearing, motor coordination, and blood pressure checked. Psychometric assessment should look at the child's verbal and non-verbal abilities, and reading achievement. The child should also be assessed for the well recognised co-morbidities of antisocial behaviour disorder, emotional disorder, tic disorder, pervasive developmental (autistic spectrum) disorder, general and specific learning difficulties, language impairment, motor planning problems, and problems with self esteem.

Such assessments require input from many different professionals. This model is not realistically achievable in most secondary district services. It will never be available for $1 \%$ of the school 
population without a major, and probably unacceptable shift, in paediatric resources, staff, and training. Anecdotal evidence suggests that although many services have tried to follow this protocol, very few are actually succeeding. The most common service deficit outside the tertiary centres is a lack of clinical psychology, which is essential for detailed psychometric testing and for home based, behaviour management. As services are currently configured, these gold standard guidelines are not available for the large numbers of families who need help. Limousines for some or public transport for all is always a stark choice.

NICE sheds more light on the cost to the English and Welsh economies of the better recognition and treatment of hyperkinesis. In 2000, $1 \%$ of the school age population represented 69000 children in England and 4200 in Wales aged 6-16. Of these, approximately 49000 children were not receiving medication. If all these children had a trial of methylphenidate, at an average dose of $30 \mathrm{mg}$, the cost of the drug alone, for both responders and non-responders

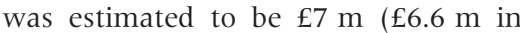
England and $£ 0.4 \mathrm{~m}$ in Wales) in the first year. Health, social services, and education would also incur costs for the assessment and follow up of these children. The costs were estimated at about $£ 23 \mathrm{~m}$ for initial specialist assessment ( $£ 21.8 \mathrm{~m}$ England, $£ 1.5 \mathrm{~m}$ Wales) and another $£ 14 \mathrm{~m}$ for follow up care in the first year. All costs are at 2000 prices. $^{3}$

The number of children on medication has risen and continues to rise. Figures from the Department of Health show that in 2002 there were approximately 208000 prescriptions for methylphenidate (Ritalin, Equasym), up from 158000 in 1999, 127000 in 1998, and 92000 in 1997.6

In the UK, the views and support of general practitioners are critical to the success of any service. In some districts, the NICE guidelines with their emphasis on specialist assessment and treatment had a negative impact on the approach of general practitioners to hyperkinesis in their practice populations. Some general practitioners became reluctant to prescribe repeat prescriptions for controlled drugs to children. Others refused completely, forcing families to attend additional clinic appointments, children to take unnecessary days off school, and waiting times for clinics to increase. The recent introduction of atomoxetine (Strattera), a noncontrolled, non-stimulant, selective noradrenaline reuptake inhibitor for hyperkinesis may resolve some of these difficulties. However, this is not yet in widespread general use and most UK specialists have only limited experience of the drug. The wide ranging Shipman Inquiry into the use of controlled drugs in the community may also impact on prescribing in the future. ${ }^{7}$

Hyperkinesis sums up many of the dilemmas that will increasingly face paediatricians. Our current services for children divide their needs artificially. Physical, educational, and emotional problems are seen as distinct entities managed in geographically separate buildings, by separate professionals, and by teams with different philosophies. Stressed and failing parents are passed from appointment to appointment, with family tensions increasing en route. Social services involvement and family breakdown will be the end point for many.

Children with hyperkinesis will not occupy acute hospital beds or figure in waiting list targets. Yet their outcomes are poor. Many will fail or be excluded from school. Others will suffer the social consequences of their impulsive behaviours in secure units or prison. Although the symptoms of hyperkinesis reduce with age, some young adults continue to need medication and supervision. It is currently almost impossible to find suitable adult services prepared to take them on.

More general paediatricians and general practitioners need to become involved in managing these children and supporting their families. More paediatricians need to take an interest in behaviour management. The "Child in Mind" initiative of the Royal College of Paediatrics and Child Health, which aims to teach paediatricians in training child mental health as it relates to paediatrics, is a promising beginning. ${ }^{8}$ This could eventually result in the development of a new type of paediatrician with a special interest in behaviour management. It is as yet a drop in the ocean compared to the unmet need. It is hoped that when NICE reviews the national guidelines with their emphasis on specialist management, they will be modified to encourage more generic professionals to become involved.

Parents also need someone who "does behaviour", and when they need help, they need it urgently. Commissioners should ensure that effective services are available, even if this involves difficult decisions for all who care for and about children.

Arch Dis Child 2005;90:222-223. doi: 10.1136/adc.2004.057406

Correspondence to: Dr M Mather, Consultant Community Paediatrician, Bexley Care Trust, UK; mary.mather@bexley.nhs.uk

\section{REFERENCES}

1 Foreman DM, Foreman D, Minty EB. The association between hyperkinesis and breakdown of parenting in clinic populations. Arch Dis Child 2005;90:245-8.

2 Buitelaar JK, van Engeland H. Epidemiological approaches. In: Sandberg S, ed. Hyperactivity disorders of childhood. Cambridge: Cambridge University Press, 1996:26-68.

3 National Institute for Clinical Excellence. Guidance on the use of methylphenidate for attention deficit/hyperactivity disorder (ADHD) in childhood. Technology appraisal guidance no. 13 . London: NICE, October 2000.

4 Scottish Intercollegiate Guidelines Network. Attention deficit and hyperactive disorders in children and young people. A national clinical guideline. Edinburgh: SIGN, Executive Royal College of Physicians, June 2001.

5 Hill P, Taylor E. An auditable protocol for treating attention deficit/hyperactivity disorder. Arch Dis Child 2001;84:404-9.

6 Department of Health. Prescription cost analysis, England 2002. London: The Stationary Office, May 2003.

7 The Shipman Inquiry, fourth report. The regulation of controlled drugs in the community. London: The Stationary Office, July 2004.

8 http://www.rcpch.ac.uk. 


\section{Respiratory support of infants with bronchiolitis related apnoea: is there a role for negative pressure?}

\author{
J Henderson
}

\section{Commentary on the paper by Al-balkhi et al (see page 288)}

$\mathrm{N}$ egative pressure ventilation is not new. Indeed the first practical ventilator for human subjects was the iron lung designed in the late 1920s to provide ventilatory assistance to patients with poliomyelitis. ${ }^{1}$ However, negative pressure ventilation has been largely superseded by developments of positive pressure ventilation equipment and techniques, including non-invasive positive pressure ventilation. In this issue of the journal, Al-balkhi and colleagues suggest a role for negative pressure ventilation in the treatment of infants with apnoea associated with bronchiolitis. $^{2}$ So is negative pressure ventilation on the brink of a comeback or should it be properly consigned to history?

Negative pressure ventilation (NPV) relies on the application of a subatmospheric pressure to the thorax, which is transmitted to a reduction of intrapleural pressure, leading to expansion of the lungs. By cycling the pressure and allowing passive deflation of the lungs as the negative intrapleural pressure rises, alveolar ventilation can occur. Advantages of negative pressure ventilation include avoidance of the adverse effects of endotracheal intubation and positive pressure ventilation. The patient's airway may be accessed readily during NPV for diagnostic or therapeutic procedures such as suctioning of secretions or fibreoptic bronchoscopy. There is also a beneficial effect on cardiac output, probably related to increased systemic venous return, although this effect may only apply when negative pressure is applied to the thorax alone using a cuirass or wrap rather than to the entire body surface as occurs with a tank ventilator. ${ }^{3}$ Negative pressure ventilation has been successfully applied to the treatment of children after cardiac surgery, particularly total cavo-pulmonary connection (Fontan) ${ }^{4}$ or correction of tetralogy of Fallot. ${ }^{5}$ Potential problems associated with NPV include augmentation of upper airway obstruction in patients with abnormalities of the extrathoracic airway or bulbar dysfunction and the possibility of reflux and aspiration associated with dynamic effects on the lower oesophageal sphincter. $^{\circ}$

Studies of the role of negative pressure ventilation in acute respiratory disorders in adult patients have focused on clinical outcomes in acute respiratory failure associated with chronic obstructive pulmonary disease (COPD) or neuromuscular disorders. These were recently reviewed by Corrado and Gorini. ${ }^{7}$ In adults with COPD, a number of uncontrolled studies have reported short term improvements in gas exchange, but the significance of these physiological changes to clinical outcomes is uncertain. A controlled study of NPV compared with conventional ventilation in adults with COPD reported no significant differences in mortality but a reduction in duration of ventilation in patients treated with NPV. ${ }^{8}$ A comparison of two different modalities of non-invasive ventilation (NPV and non-invasive positive pressure ventilation) reported similar proportions that needed rescue invasive ventilation and no significant difference in mortality between treatment groups. However, patients treated with NPV had significantly shorter duration of ventilation and length of hospital stay. ${ }^{9}$ Therefore, there are data from the adult literature to suggest a beneficial effect of NPV on physiological and clinical outcomes in acute on chronic respiratory failure associated with COPD. However, this is a very different population from infants and young children with acute respiratory failure. In the paediatric population, negative pressure ventilation has been reported to be effective in the management of neonatal acute respiratory distress syndrome in uncontrolled $^{1011}$ and controlled studies. ${ }^{1213}$ Samuels and Southall have previously reported short term reductions in oxygen requirements of infants and young children with respiratory failure due to a variety of causes who were treated with $\mathrm{NPV}^{14}$ and, in a randomised controlled trial of treatment of neonatal respiratory distress syndrome, Samuels and colleagues reported that continuous negative extrathoracic pressure (CNEP) reduced the need for endotracheal intubation and its subsequent pathophysiological consequences of prolonged oxygen requirement and chronic lung disease compared with standard therapy. ${ }^{15}$ Outside the neonatal age group, there are few published randomised controlled studies of the use of NPV in the treatment of acute respiratory failure. A recent Cochrane systematic review identified one study, published in abstract form only, that met adequate methodological criteria. ${ }^{16}{ }^{17}$ This study reported short term reduction in oxygen requirements in a proportion of infants with bronchiolitis treated with CNEP. However, the Cochrane systematic review concluded that there was insufficient evidence on which to judge the effectiveness of CNEP. The authors reached the same conclusion about non-invasive continuous positive airway pressure (CPAP) treatment of children with acute hypoxaemic respiratory failure. The apparent lack of a sound evidence base for this commonly applied treatment modality is perhaps surprising. A nonrandomised study has reported short term improvements in physiological variables in infants with bronchiolitis and "impending respiratory failure" treated with CPAP, ${ }^{18}$ but there has been no systematic evaluation of this treatment in the paediatric age group.

So what does the study by Al-balkhi and colleagues add? The entry criterion was bronchiolitis related apnoea, a common reason to consider ventilator assistance in infants but one about which relatively little is understood in terms of its mechanisms. It is suggested that respiratory syncytial virus (RSV) may augment laryngeal chemoreceptor responses ${ }^{19}$ and similarities exist between reflex laryngeal apnoea, apnoea of prematurity, and that associated with RSV. ${ }^{20}$ Alternatively the association of RSV related apnoeas with preterm infants or those of young postnatal age points to effects on immature respiratory control mechanisms. ${ }^{21}$ Apnoeas tend to occur early in the course of RSV bronchiolitis and have been reported to be associated with a significant risk of mechanical ventilation, with the relative risk increasing from 2.4 for one apnoea to 6.5 for multiple apnoeas in one large study. ${ }^{22}$ Therefore, the study population appears to be appropriate to the question posed. The retrospective design of this study and the relatively small number of subjects studied were limitations acknowledged by the authors, and the absence of key data, such as gas exchange at entry, makes true comparison of disease 
severity between the study populations difficult. Although "standard" treatment was used in both study centres, there was a significant difference in the proportions of infants treated with methylxanthines. Methylxanthines have been reported to increase diaphragmatic contractility ${ }^{23}$ and a combined effect of this with the inhibition of inspiratory muscle activity by $\mathrm{NPV}^{24}$ on efficiency of ventilation cannot be discounted, in addition to the reported effects of caffeine on RSV related apnoea cited by the authors. ${ }^{25}$ However, the authors have not been extravagant in their claims for negative pressure ventilation in this context and have produced some interesting, although preliminary findings. Their call for a prospective randomised controlled trial of respiratory support strategies in the treatment of bronchiolitis related apnoea is reasonable. The design of such a study should include clinically important as well as physiological outcomes. ${ }^{26}$ However, given the well publicised curtailment of activities of one of the principal research active groups in this field, it may be some time before such a trial sees the light of day.

Where does this leave the clinician caring for an infant with bronchiolitis and clinically significant apnoeas? Despite the lack of evidence from randomised control trials, it would appear that non-invasive respiratory support offers the potential advantage of avoiding endotracheal intubation, at least for some infants. The majority of institutions caring for infants and young children with critical respiratory illnesses will have access to equipment and expertise for the delivery of non-invasive CPAP and/or bilevel positive airway pressure (BiPAP). Until further evidence becomes available, this modality is likely to remain the first choice therapeutic intervention for infants with bronchiolitis related apnoea.

Arch Dis Child 2005;90:224-225.

doi: $10.1136 /$ adc. 2004.054007

Correspondence to: Dr J Henderson, University of Bristol, Royal Hospital for Children, Paul $\mathrm{O}^{\prime}$ Gorman Building, Department of Respiratory Medicine, Upper Maudlin Street, Bristol BS2

8BJ, UK; a.j.henderson@bris.ac.uk

\section{REFERENCES}

1 Drinker PA, McKhann CF. Landmark perspective: The iron lung. First practical means of respiratory support. JAMA 1986;255:1476-80.

2 Al-balkhi A, Klonin H, Marinaki K, et al. Review of treatment of bronchiolitis related apnoea in two centres. Arch Dis Child 2005;90:288-91.

3 Lockhat D, Langleben D, Zidlka A. Hemodynamic differences between continuous positive and two types of negative pressure ventilation. Am Rev Respir Dis 1992;146:677-80.

4 Shekerdemian LS, Bush A, Shore DF, et al. Cardiorespiratory interaction after Fontan operations: augmentation of cardiac output using negative pressure ventilation. Circulation 1997:96:3934-42

5 Shekerdemian LS, Bush A, Shore DF, et al. Cardiorespiratory responses to negative pressure ventilation after tetralogy of Fallot repair: a hemodynamic tool for patients with a low-output state. J Am Coll Cardiol 1999;33:549-55.

6 Marino WD, Pitchumoni CS. Reversal of negative pressure ventilation-induced lower esophageal sphincter dysfunction with metoclopramide. J Gastroenterol 1992;87:190-4.

7 Corrado A, Gorini M. Negative pressure ventilation: is there still a role? Eur Respir $J$ 2002; 20: 187-97.

8 Corrado A, Gorini M, Ginanni R, et al. Negative pressure ventilation versus conventional mechanical ventilation in the treatment of acute respiratory failure. Eur Respir J 1998; 12:519-25.

9 Corrado A, Confalonieri M. Marchese S, et al. Iron lung versus mask ventilation in the treatmen of acute on chronic respiratory failure in COPD patients: a prospective randomised controlled study. Chest 2002;121:189-95.

10 Outerbridge EW, Roloff DW, Stern L. Continuous negative pressure in the management of severe respiratory distress syndrome. J Pediatr 1972;81:384-91.

11 Alexander G, Gerhardt T, Brancalari E. Hyaline membrane disease. Comparison of continuous negative pressure and nasal positive airway pressure in its treatment $A m$ J Dis Chest 1979; 133:1156-9

12 Fanaroff AA, Cha CC, Sosa R, et al. Controlled trial of continuous negative external pressure in the treatment of severe respiratory distress syndrome. J Pediatr 1973;82:921-8.

13 Silverman WA, Sinclair JC, Gandy GM, et al. A controlled trial of management of respiratory distress syndrome in a body-enclosing respirator. 1. Evaluation of safety. Pediatrics 1967;39:740-8.

14 Samuels MP, Southall DP. Negative extrathoracic pressure in the treatment of respiratory failure in infants and young children. BMJ 1989;299: 1253-7.

15 Samules MP, Raine J, Wright T. Continuous negative extrathoracic pressure in neonatal respiratory failure. Pediatrics 1996;98:1154-60

16 Shah PS, Ohlsson A, Shah JP. Continuous negative extrathoracic pressure or continuous positive airway pressure for acute hypoxemic respiratory failure in children (Cochrane review) In: The Cochrane Library, Issue 1. Chichester: John Wiley \& Sons, 2004.

17 Hartman H, Noyes JP, Wright T, et al. Continuous negative pressure ventilation in infants with bronchiolitis. Eur Respir J 1994;S18:379.

18 Soong WJ, Hwang B, Tang RB. Continuous positive pressure by nasal prongs in bronchiolitis. Pediatr Pulmonol 1993;16:163-6.

19 Lindgren C, Grogaard J. Reflex apnoea response and inflammatory mediators in infants with respiratory tract infection. Acta Paediatr 1996:85:798-803

20 Pickens DL, Schefft GL, Storch GA, et al. Characterization of prolonged apneic episodes associated with respiratory syncytial virus infection. Pediatr Pulmonol 1989;6:195-201.

21 Church NR, Anas NG, Hall CB, et al. Respiratory syncytial virus-related apnea in infants. Demographics and outcome. Am J Dis Child 1994; 138:247-50.

22 Kneyber MCJ, Brandenburg $\mathrm{AH}$, de Groot $\mathrm{R}$, et al. Risk factors for respiratory syncytial virus associated apnoea. Eur J Paediatr 1998; 157:331-5

23 Aubier M, Murciano D, Viires N, et al. Diaphragmatic contractility enhanced by aminophylline: role of extracellular calcium. J Appl Physiol 1983;54:460-4.

24 Glerant J-C, Jounieaux V, Parreira VF, et al. Effects of intermittent negative pressure ventilation on effective ventilation in normal awake subjects. Chest 2002;122:99-107.

25 Tobias JD. Caffeine in the treatment of apnea associated with respiratory syncytial virus infection in neonates and infants. South Med J 2000;93:294-6.

26 Randolph AG, Meert KL, O'Neil ME, et al. The feasibility of conducting clinical trials in infants and children with acute respiratory failure. $\mathrm{Am}$ Rev Respir Crit Care Med 2003;167:1334-40. 